Supporting Information

\title{
Engineering of Annealing and Surface Passivation toward Efficient and Stable Quasi-2D Perovskite Light Emitting Diodes
}

Yunxing Fu ${ }^{\mathrm{a}, \mathrm{b}}$, Dezhong Zhang ${ }^{\mathrm{a}}$, Hongmei Zhan ${ }^{\mathrm{a}}$, Chenyang Zhao ${ }^{\mathrm{a}}$, Yanxiang Cheng,

Chuanjiang Qin ${ }^{\mathrm{a}, b^{*}}$, Lixiang Wang ${ }^{\mathrm{a}}$

aState Key Laboratory of Polymer Physics and Chemistry, Changchun Institute of Applied Chemistry, Chinese Academy of Sciences, Changchun 130022 (P. R. China).

bShool of Applied Chemistry and Engineering, University of Science and Technology of China, Hefei 230026 (P. R. China).

*E-mail: cjqin@ciac.ac.cn 


\section{Methods}

\section{Materials}

PVK, PEABr, FABr, $\mathrm{PbBr}_{2}$ and $\mathrm{MACl}$ were purchased from Xi'an Polymer Light Technology Corp. TPBi and LiF were purchased from Jilin Oled Material Technology Corp. Trimethylolpropane trimethacrylate was purchased from Energy-Chemical Corp. DMF, DMSO, and ethylacetate were purchased from J\&K Scientific Corp. All the chemical materials were directly used without any further purifications.

\section{Preparation of quasi-2D perovskite layers}

The quasi-two-dimensional perovskite precursor is configured by dissolving $\mathrm{PEABr}, \mathrm{FABr}, \mathrm{PbBr}_{2}$ and $\mathrm{MACl}$ in DMSO at a stoichiometric ratio of 2:4:5:0.5, and the MACl acts as additive to improve the crystallization. The concentration of $\mathrm{PbBr}_{2}$ is $0.2 \mathrm{M}$. After the solute is completely dissolved, $0.45 \mu \mathrm{m}$ organic filter is used to filter the solution. The precursor solution is spin-coated at $7000 \mathrm{rpm}$ for $30 \mathrm{~s}$, and then $100 \mu \mathrm{L}$ of ethyl acetate is poured onto the film at the 10th second, followed by annealing on a hot plate at $85^{\circ} \mathrm{C}$. For the TPTA modified film, TPTA is added to ethyl acetate at a concentration of 2 $\mathrm{mM}$.

\section{Light-Emitting Diodes Fabrication}

The indium tin oxide (ITO)-coated glass substrates are sequentially cleaned in detergent, distilled water, acetone and isopropanol by an ultrasonic cleaner. The pre-cleaned substrates are ultraviolet ozone treated for $30 \mathrm{~min}$ to make the surface hydrophilic, and then transferred into a nitrogen-filled glove box. PVK solution (10 $\mathrm{mg} \mathrm{mL}^{-1}$ in chlorobenzene) is spin-coated at $4000 \mathrm{rpm}$ for $40 \mathrm{~s}$ and the films are baked at $120{ }^{\circ} \mathrm{C}$ for $30 \mathrm{~min}$. After that, the perovskite film is deposited on PVK layer. Ultimately, TPBi $(50 \mathrm{~nm}), \operatorname{LiF}(1 \mathrm{~nm})$, and $\mathrm{Al}(100 \mathrm{~nm})$ are deposited by thermal evaporation, respectively. The active device area is $0.08 \mathrm{~cm}^{2}$

\section{Characterization}

UV-Vis absorption spectra of quasi-2D perovskite layers were obtained by a Perkin-Elmer Lambda 35 UV-vis spectrometer. The GIXRD measurements were performed at Shanghai Synchrotron Radiation 
Facility using X-ray with a wavelength of $1.24 \AA$ and an incidence angle of $0.13^{\circ}$. PL spectra and PLQY were measured by a HORIBA FL3C-111 spectrofluorometer equipped with an integrating sphere, and the excitation wavelength is $385 \mathrm{~nm}$ from a xenon lamp light source. The transient PL-decay curves were measured with an Edinburgh FLS-980 fluorescence spectrophotometer with an excitation source of a $390 \mathrm{~nm}$ laser. Topography images of the corresponding films were collected by SPI3800N AFM. XRD curve was obtained using a Bruker D8 diffractometer with a wavelength of 1.54 $\AA$ and an incidence angle of $0.2^{\circ}$. All the PeLEDs performance tests were performed with an Ocean Optics LED integrating sphere test system in glove box. 

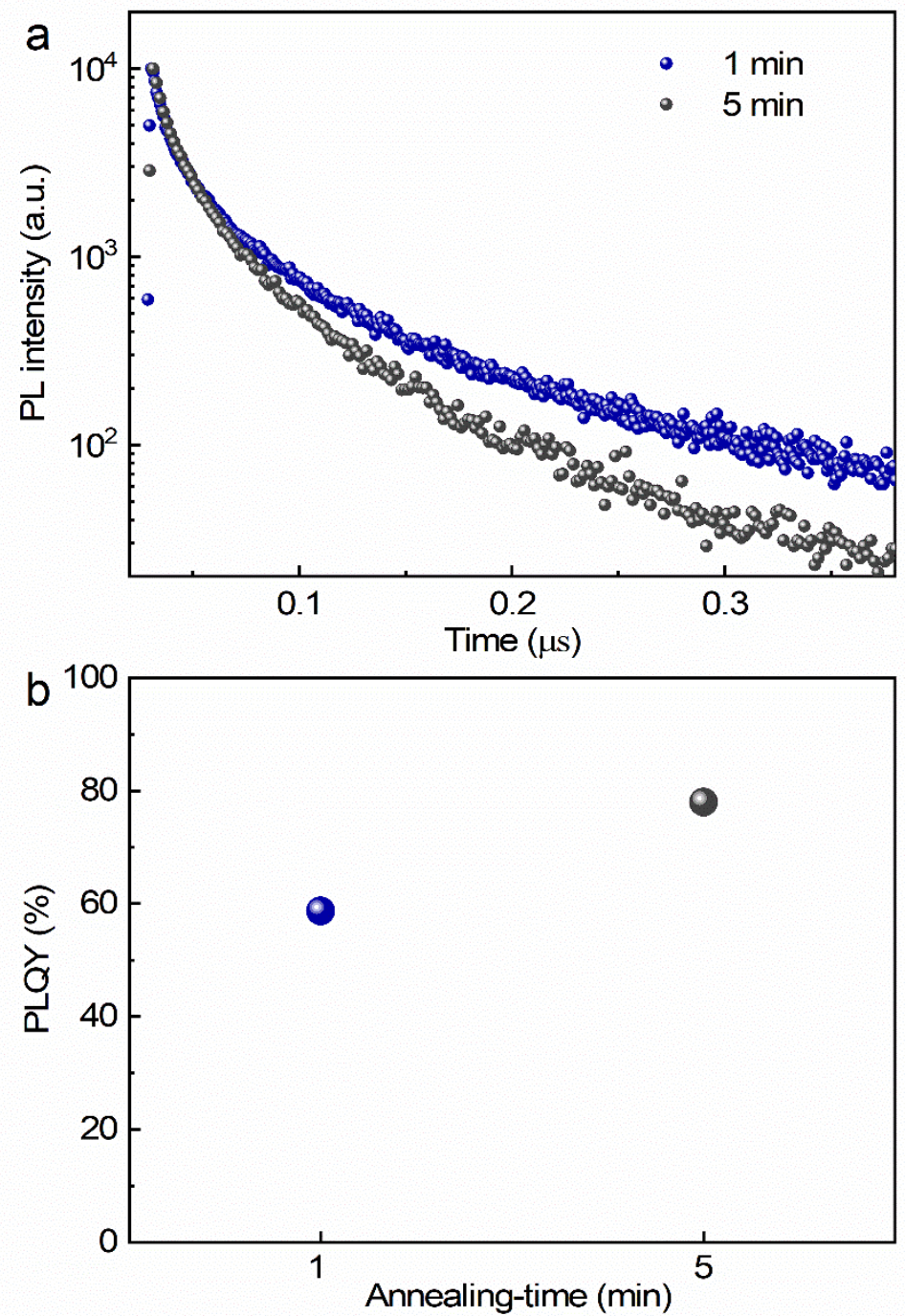

Figure S1. a) Transient fluorescence decay and b) PLQY of quasi-2D perovskite layer obtained by annealing for $1 \mathrm{~min}$ and $5 \mathrm{~min}$. 

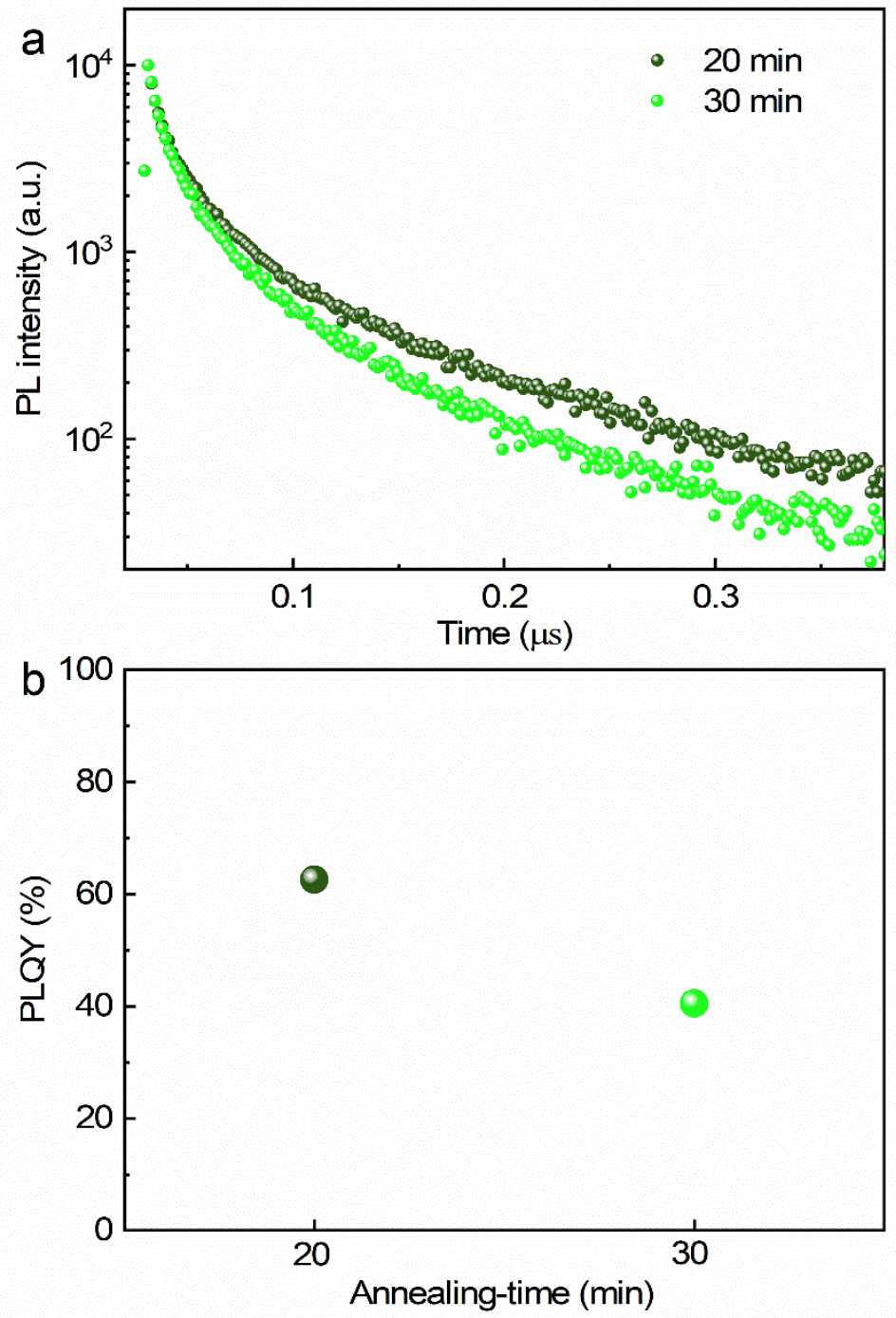

Figure S2. a) Transient fluorescence decay and b) PLQY of quasi-2D perovskite layer obtained by annealing for $20 \mathrm{~min}$ and $30 \mathrm{~min}$. 

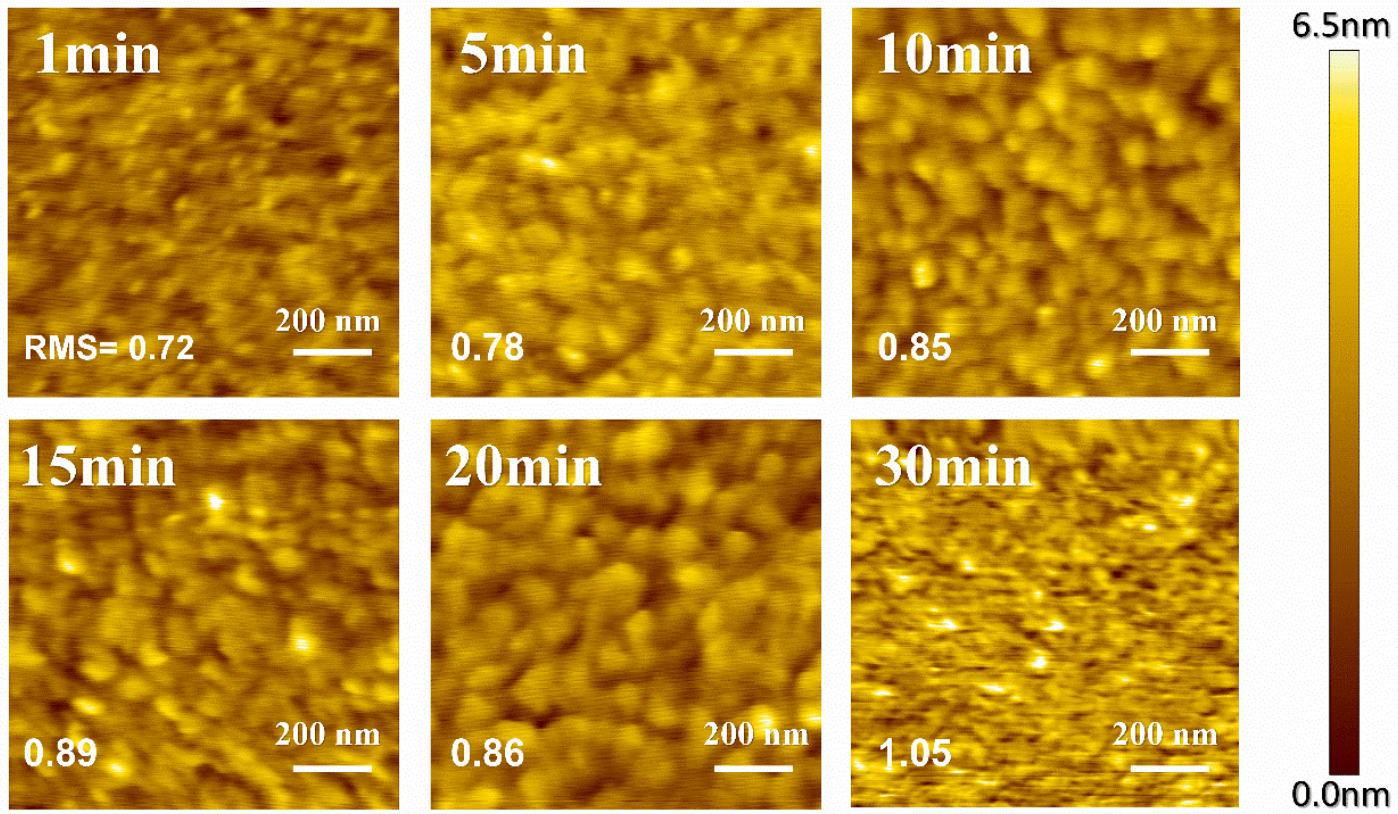

Figure S3. AFM images of quasi-2D perovskite films by annealing for different time. 


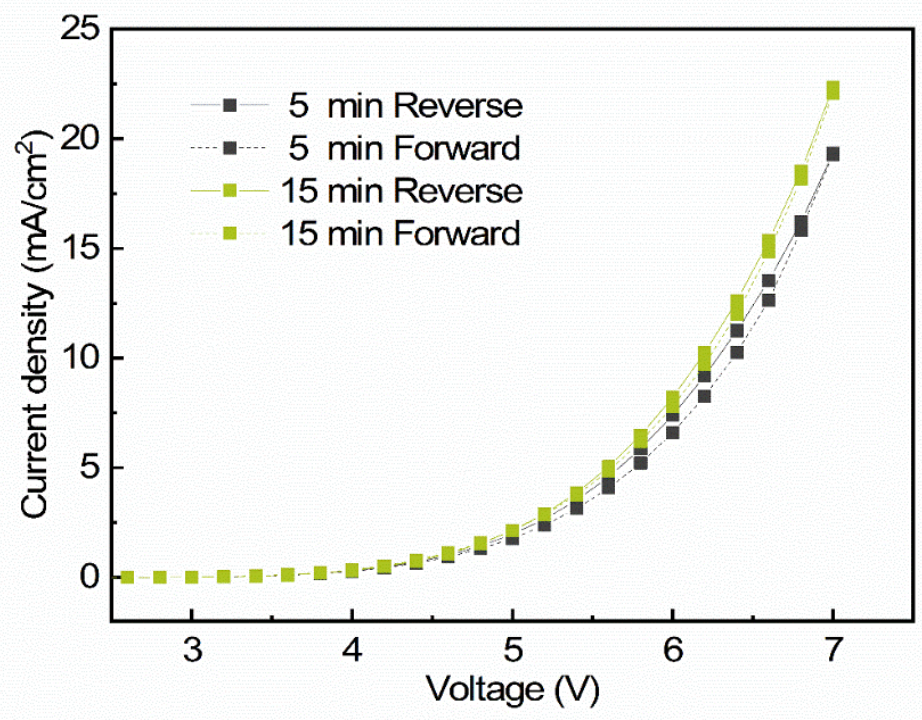

Figure S4. Current density-voltage hysteresis of PeLEDs prepared by different perovskite annealing time. 

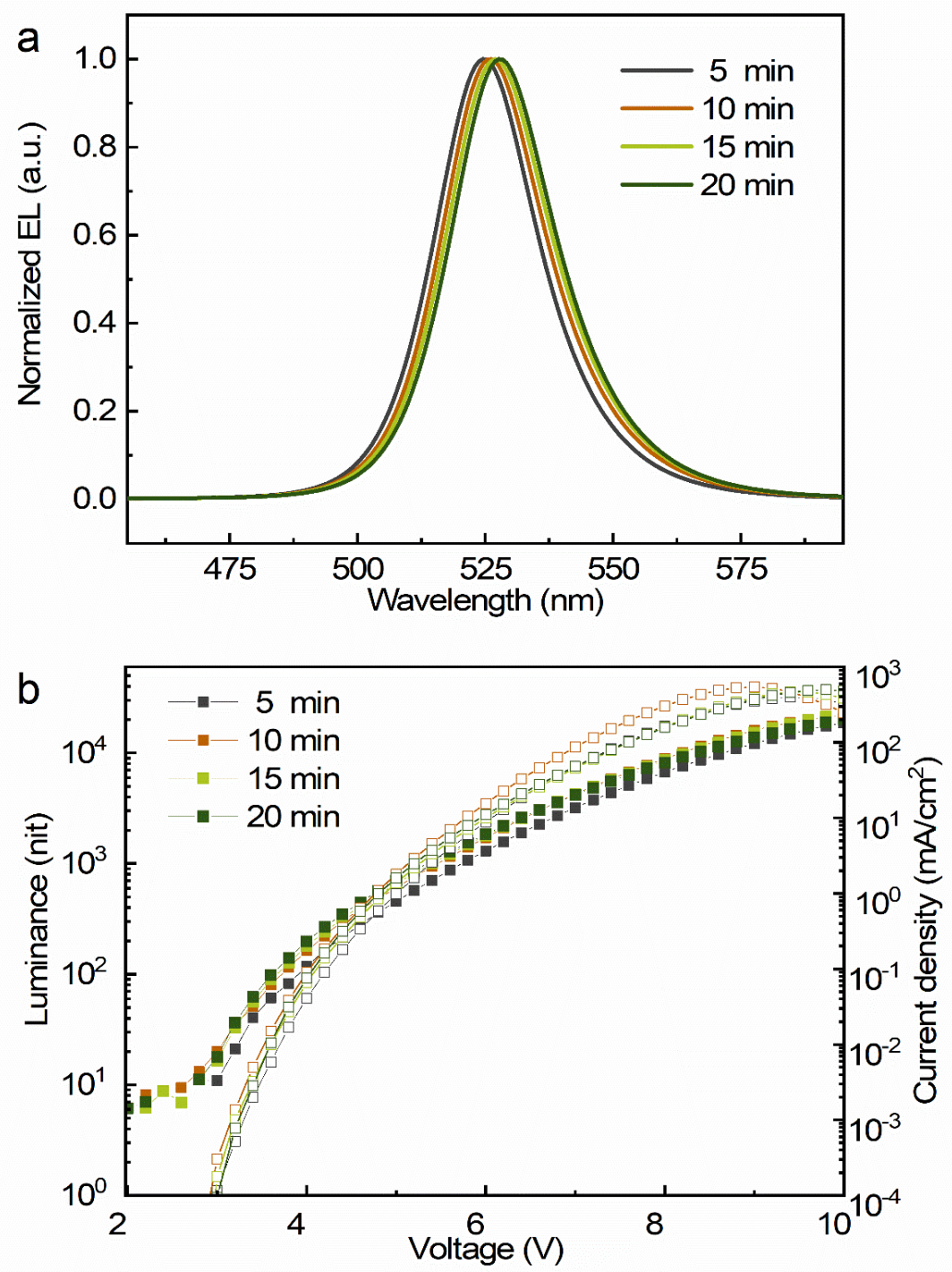

Figure S5. a) EL spectra of quasi-2D PeLEDs under the identical bias. b) Luminance-voltage-current density characteristics of fabricated LED devices. 


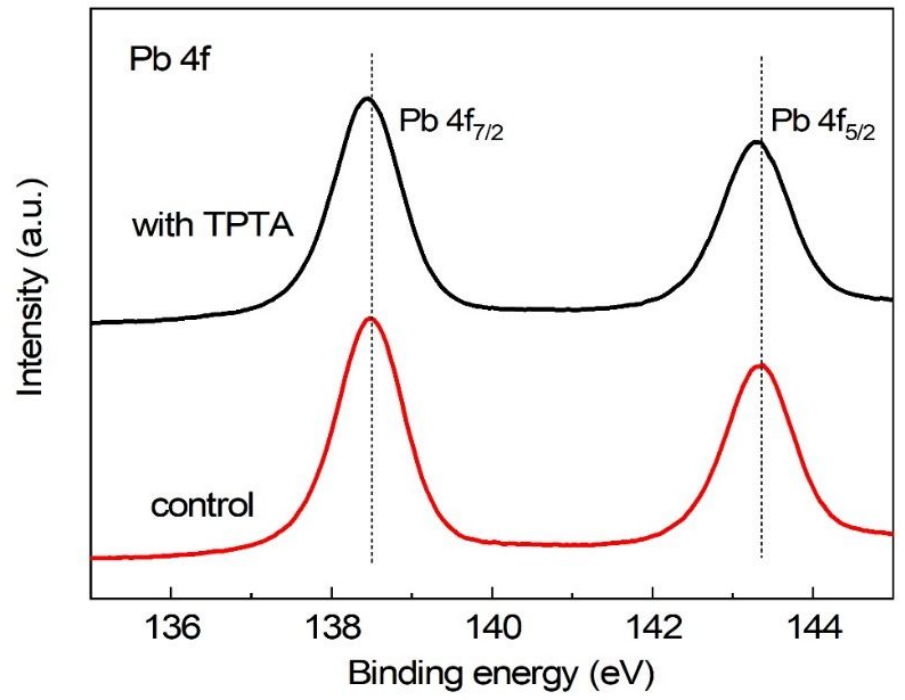

Figure S6. XPS spectra of the control and TPTA-treated films for $\mathrm{Pb} 4 \mathrm{f}$ orbit. 

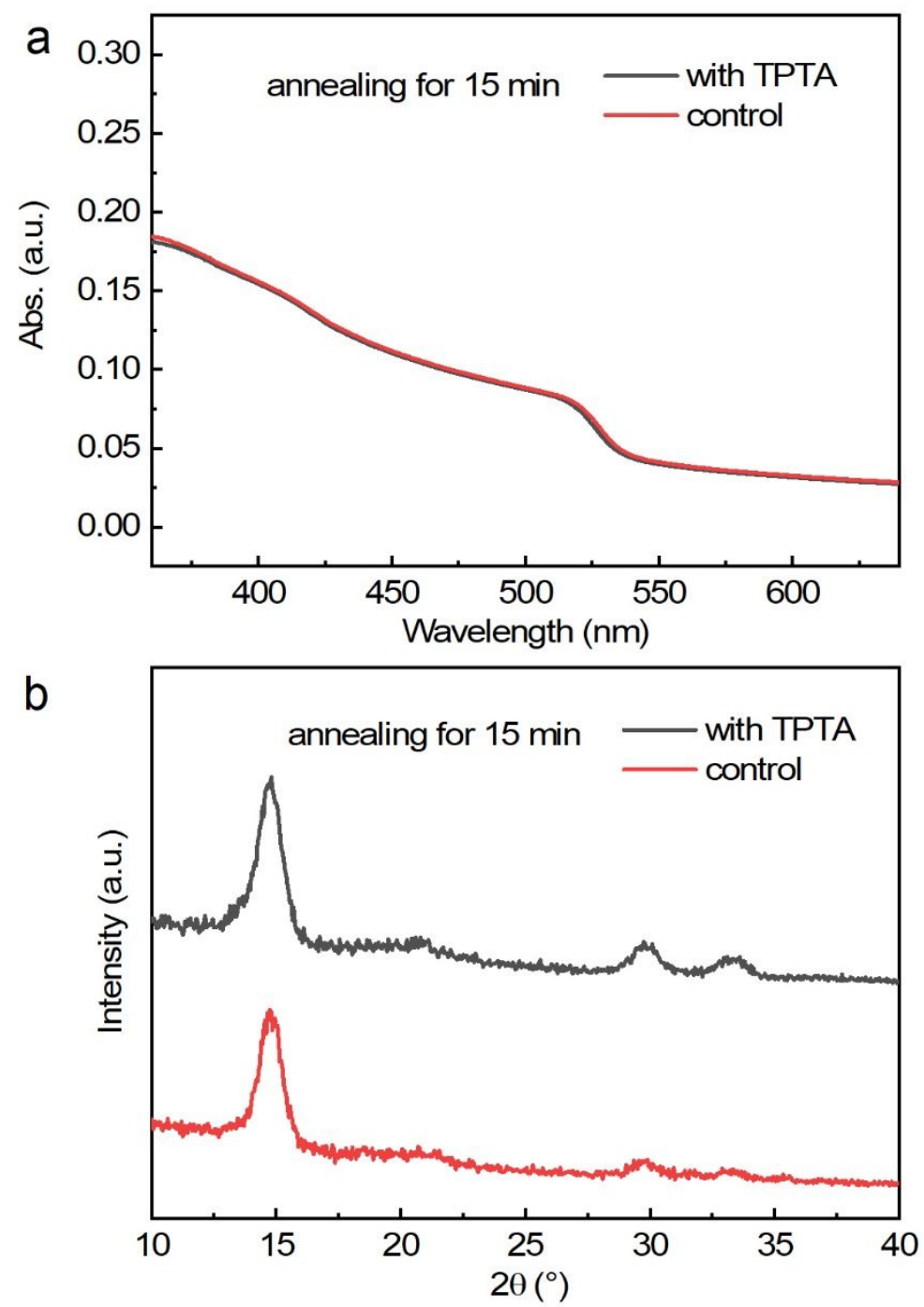

Figure S7. a) UV-Vis absorption spectra and b) XRD patterns of quasi-2D perovskite layer with or without TPTA. 

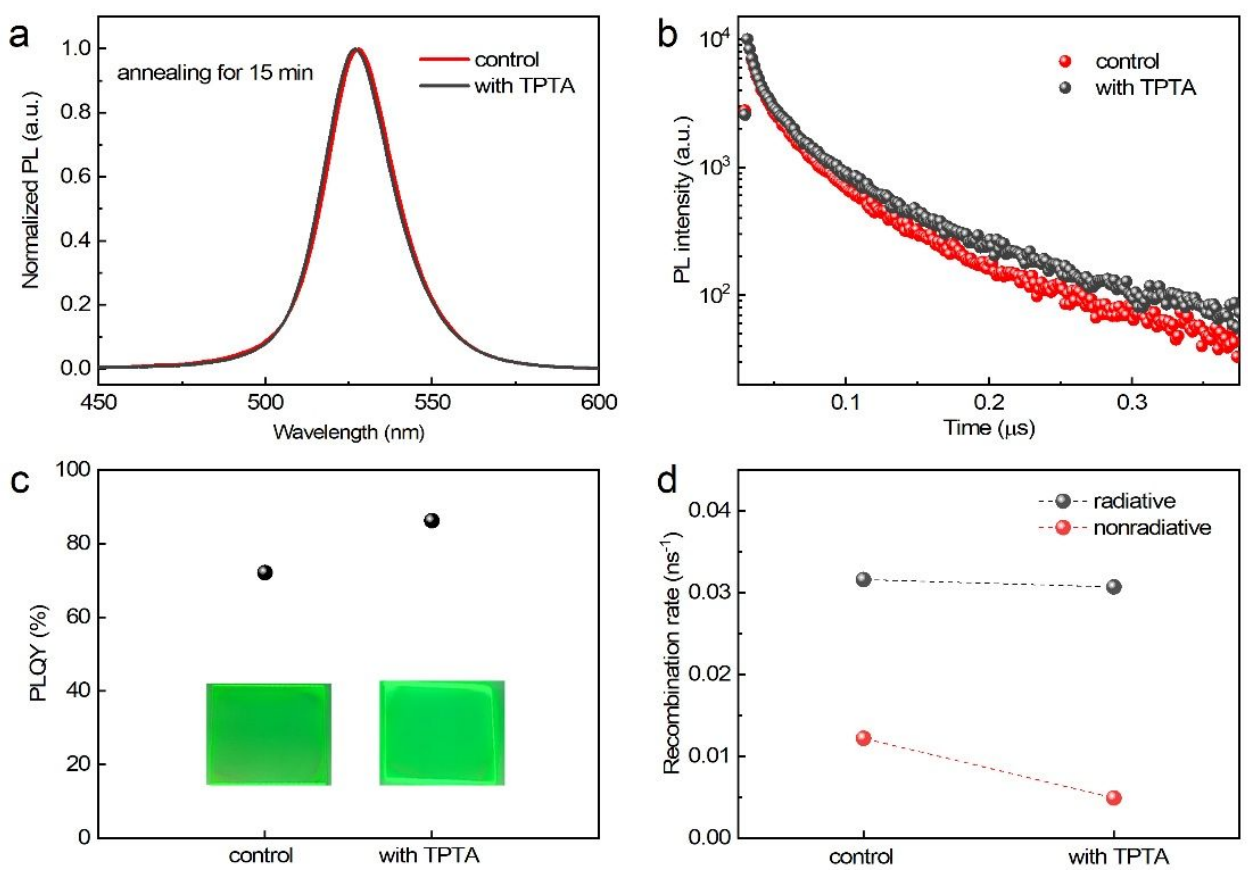

Figure S8. a) Steady-state PL, b) transient PL, c) PLQY, and d) radiative and nonradiative recombination rate of quasi-2D perovskite films with or without TPTA after annealing for $15 \mathrm{~min}$.

Table S1. Summary of optical parameters of quasi-2D perovskite films with or without TPTA annealing for $15 \mathrm{~min}$.

\begin{tabular}{cccccc}
\hline Sample & Wavelength $(\mathrm{nm})$ & $\tau(\mathrm{ns})$ & PLQY $(\%)$ & $k_{\mathrm{r}}\left(\mathrm{ns}^{-1}\right)$ & $k_{n \mathrm{r}}\left(\mathrm{ns}^{-1}\right)$ \\
\hline Control & 527 & 22.8 & 72.1 & 0.0316 & 0.0122 \\
With TPTA & 527 & 28.0 & 86.2 & 0.0307 & 0.0049 \\
\hline
\end{tabular}




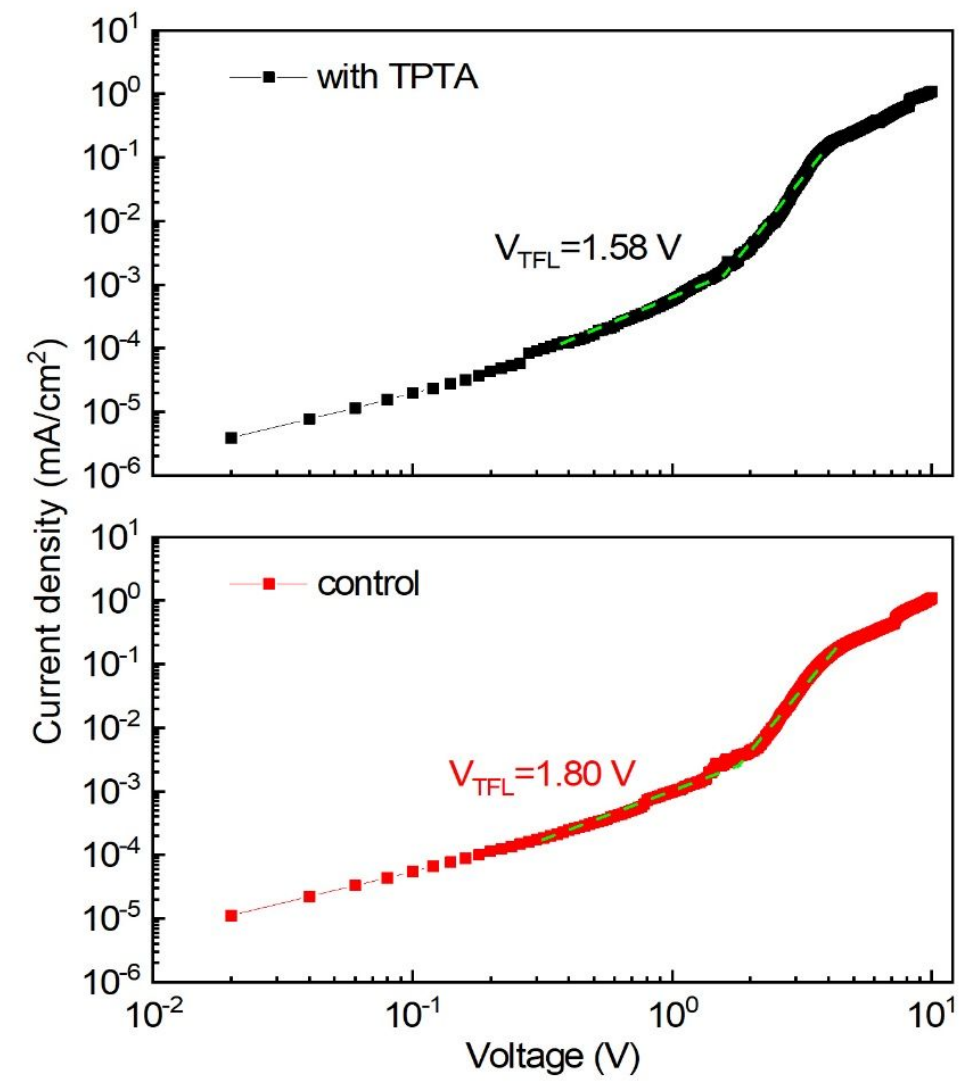

Figure S9. Space-charge-limited current (SCLC) measurements of hole-only devices with structure of ITO/PVK/perovskite/ $\mathrm{MoO}_{3} / \mathrm{Ag}$. 


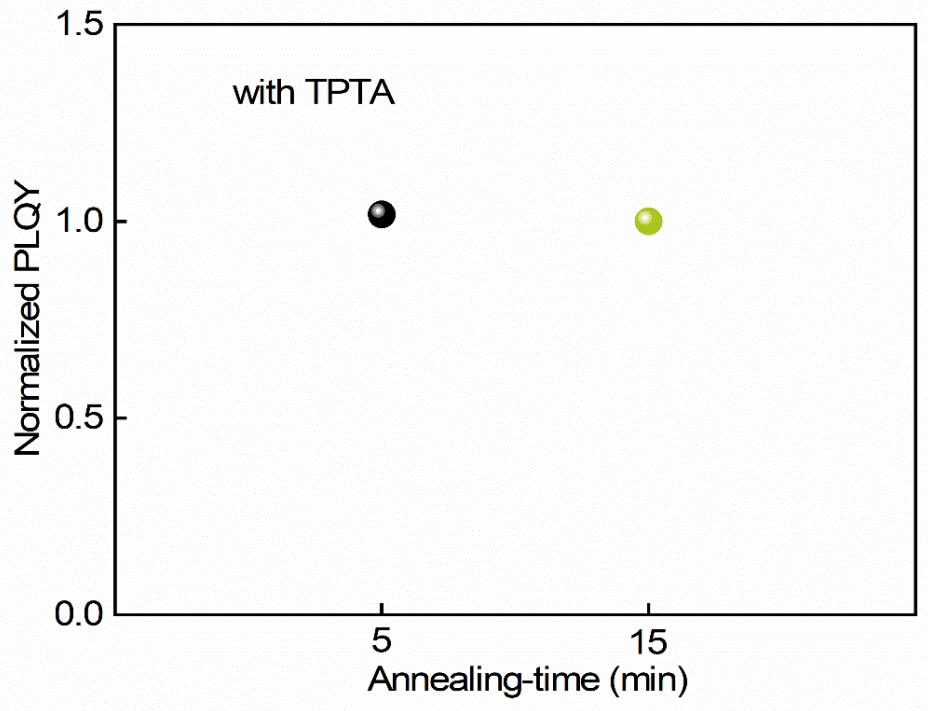

Figure S10. PLQY of quasi-2D perovskite layer obtained by annealing for $5 \mathrm{~min}$ and $15 \mathrm{~min}$ with TPTA. 

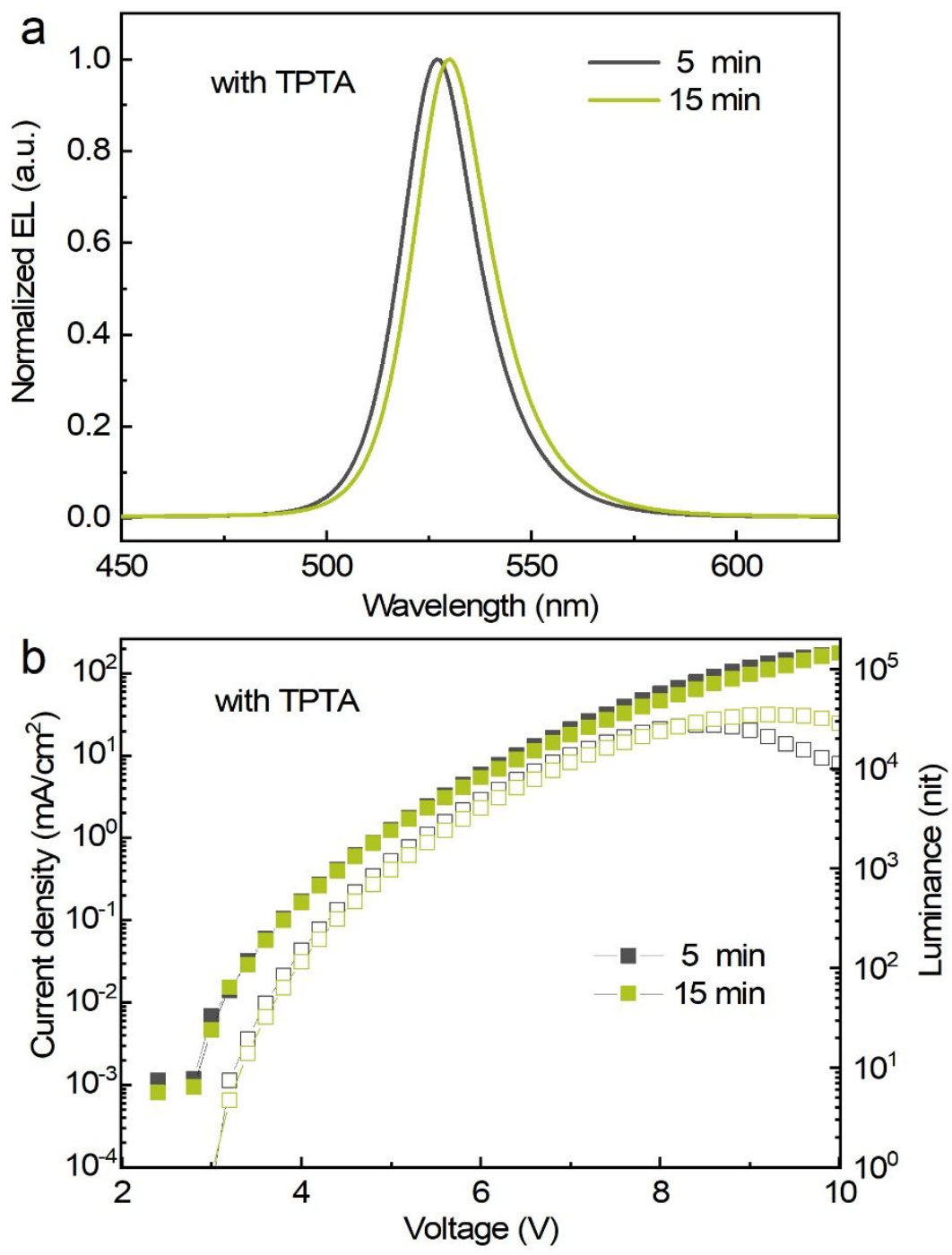

Figure S11. a) EL spectrum of TPTA-treated quasi-2D PeLEDs under the identical bias. b) Luminance-voltage-current density characteristics of TPTA-treated LED devices. 
Table S2. Summary of device performance with or without TPTA.

\begin{tabular}{ccccc}
\hline Device & $\mathrm{V}_{\text {on }}(\mathrm{V})$ & $\mathrm{L}_{\max }\left(\mathrm{cd} \mathrm{m}^{-2}\right)$ & $\mathrm{EQE}_{\text {ave }}(\%)$ & Wavelength $(\mathrm{nm})$ \\
\hline Control, 5min & 3.0 & 31931 & 17.7 & $526-523$ \\
Control, 10min & 3.0 & 39400 & 15.7 & $530-526$ \\
Control, 15min & 3.0 & 35713 & 14.0 & $530-526$ \\
Control, 20min & 3.0 & 37244 & 12.6 & $530-526$ \\
With TPTA, 5min & 3.0 & 27687 & 18.1 & $527-525$ \\
With TPTA, 15min & 3.0 & 35025 & 18.2 & $530-527$ \\
\hline
\end{tabular}

